Chinese Journal of Organic Chemistry

NOTE

\title{
多肽酰肼法合成翻译后修饰物蛋白 NEDD8
}

\author{
管超建＼cjkstart王 涛 王 君* 李宜明*
}

(合肥工业大学生物与医学工程学院 合肥 230009)

\begin{abstract}
摘要 NEDD8(神经前体细胞表达发育下调蛋白 8)是真核生物体内一种重要的类泛素化蛋白, 参与调控细胞内一系列 重要的生命过程. 目前获得 NEDD8 的主要方法是生物重组表达, 该策略的缺点在于表达量不高且需要除去外加纯化 标签的步骤. 本研究结合高温辅助固相合成技术与基于多肽酰胖连接的一锅连接-脱硫策略, 较为高效地获得了均一性 的 NEDD8 蛋白, 为进一步研究 NEDD8 修饰蛋白的结构与功能提供了基础.

关键词 NEDD8; 高温辅助固相合成; 多肽酰胖连接; 一锅连接脱硫
\end{abstract}

\section{Synthesis of Post-translational Modifier Protein NEDD8 via Ligation of Peptide Hydrazides}

\author{
Guan, Chaojian \\ Wang, Tao \\ Wang, Jun* \\ Li, Yiming* \\ (Department of Pharmacy, School of Biological and Medical Engineering, Hefei University of Technology, Hefei 230009)
}

\begin{abstract}
As an important ubiquitin-like modifier protein in eukaryotic organisms, NEDD8 (neural precursor cell expressed developmentally down-regulated 8 ) is involved in regulating a series of important life processes in cells. Nowadays, the major method for obtaining NEDD8 is recombinant protein expression. However, the yield is relatively low and the recombined tag for purification needs to be removed in an extra step. In present work, NEDD8 protein was first synthesized in homogeneity by using high temperature assisted solid-phase peptide synthesis (SPPS) combining with the one-pot ligation-desulfurization strategy. This method lays the foundation for the study of NEDD8 modified proteins in future.
\end{abstract}

Keywords NEDD8; high temperature assisted SPPS; ligation of peptide hydrazide; one-pot ligation-desulfurization

蛋白质翻译后修饰在真核生物的生命活动中发挥 着关键的作用. 作为最为重要的一类翻译后修饰, 泛素 化(Ubiquitination, Ub)参与了真核细胞内包括靶蛋白降 解、增殖与分化、DNA 损伤修复等大部分生命进程 ${ }^{[1]}$. 近 期, 一些类泛素化蛋白[如 SUMO (Small Ubiquitin-like Modifier), NEDD8 (Neural Precursor Cell Expressed Developmentally Down-Regulated 8)等]修饰在真核生物体 内也被陆续被发现. 其中 NEDD8 的结构与 Ub 最为相 似, 二者的氨基酸序列的相似性达到了 $59 \%{ }^{[2]}$. 与 $\mathrm{Ub}$ 化 过程类似, NEDD8 也是通过激活酶 E1、结合酶 E2 和连 接酶 E3 的协同作用, 使得 C 端的 Gly 共价结合到底物 蛋白的 Lys 上来发挥作用, 该过程被称为底物蛋白的 NEDD 化修饰 ${ }^{[3]}$.

2004 年, 研究人员最早在泛素 E3 酶 Cullin 家族上 发现了 NEDD 化修饰 ${ }^{[4]}$. 研究表明当 NEDD8 共价结合
至 Cullin 蛋白上后，会使 Cullin 的构象发生改变、活性 增强, 从而能够更加高效地将 $\mathrm{Ub}$ 带到底物蛋白上完成

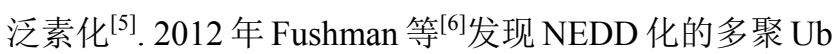
链能够终止自身链的延长, 从而维持细胞内自由 $\mathrm{Ub}$ 的 正常水平. 除了 Cullin 与多聚 Ub 链外, 人们也在细胞内 的一些其他蛋白如 Pvhl、p53 及其 E3 连接酶 Mdm2、

核糖体蛋白 L11 以及 Caspase-7 上发现了 NEDD 化修 饰 ${ }^{[7]}$. 为了进一步研究蛋白质 NEDD 化引发的生化、结 构机制，获得大量、性质均一的 NEDD8 及多聚 NEDD8 链就显得尤为重要.

前人主要通过生物重组表达的方法来获得 NEDD $8^{[8]}$. 然而使用大肠杆菌体系表达 NEDD8 的效率 较低 ${ }^{[9]}$, 同时, 为了纯化蛋白需要融入 His-tag 或者 GST 标签, 然后再使用特异性的酶来切除. 这样的策略较为 繁琐，且进一步降低了 NEDD8 的获取效率. 近年来, 随

\footnotetext{
*E-mail: wangjun-08@126.com; 1ym2007@mail.ustc.edu.cn

Received May 10, 2016; revised June 8, 2016; published online July 7, 2016.

Project supported by the National Natural Science Foundation of China (Nos. 21372058, 21572043).

国家自然科学基金(Nos. 21372058, 21572043 )资助项目.
} 
着多肽/蛋白质固相合成(Solid-phase Peptide Synthesis, SPPS)及连接技术的不断改进, 化学合成已成为获得翻 译后修饰蛋白的一种重要的手段 ${ }^{[10]}$. 本研究尝试使用 多肽酰肼连接并结合一锅连接脱硫策略合成性质均一 的 NEDD $8^{[11]}$. NEDD8 含有 76 个氨基酸, 我们首先将其 分成三片段合成并通过两次连接后获得了全长蛋白. 由 于多次分离、纯化致使合成效率降低, 我们进一步使用 高温辅助 Fmoc-SPPS 策略合成了长达 56 个 AA 的片 段 ${ }^{[12]}$, 并通过一锅连接脱硫获得了全长的 $\mathrm{NEDD} 8^{[13]}$. 最后我们通过质谱、SDS-PAGE、CD (Circular Dichroism) 等分析手段对合成蛋白进行了表征, 证明其拥有与天然 表达 NEDD8 的类似结构. 本工作为高效获得性质均一 的 NEDD8 及其他类泛素蛋白提供了一种途径, 也为研 究蛋白质 NEDD 化相关的生化、结构机制提供了基础.

\section{1 结果与讨论}

\section{1 三片段合成 NEDD8}

\subsection{1 合成 NEED8 片段 1a, 1b 与 1c}

通过对 NEDD8 的序列分析我们发现整个片段只有 两个 Ala $(56,57$ 位)适合作为连接位点. 如果使用两片 段合成则第一片段将会含有 56 个 AA, 而超过 50 个氨 基酸的长片段多肽通常难以合成或合成效率低下 ${ }^{[14]}$.
因此，我们首先尝试采用三片段连接策略合成 NEDD8 (图 1). 由于 $1 \mathbf{a}$ 片段的 Gly35 与 Ala 在结构上仅相差一 个亚甲基，且该氨基酸并不处于蛋白质的关键位点. 因 此, 选择 Gly35 为连接位点, 首先将其用 Cys 替代, 在接 后再脱硫即可获得 Ala35, 最终通过三片段连接合成 NEDD8. 使用常规的 Fmoc 固相合成方法获得了三个多 肽片段 1a, 1b, 1c. 为了防止 Met 氧化形成副产物, 在合 成中使用 Nle 代替片段中的 $\mathrm{Met}^{[15]}$. 经半制备 HPLC (High Performance Liquid Chromatography)分离后得到 纯品 $1 \mathrm{a} 35.4 \mathrm{mg}$ (分离产率 19.9\%), 1 b $31.4 \mathrm{mg}$ (分离产率 $37.3 \%$ ), 1c $18.6 \mathrm{mg}$ (分离产率 30.5\%)(图 2). 色谱、质谱 分析证明了三段多肽合成的正确性.

\subsection{2 多肽酰胖法连接片段 $\mathbf{1 a}$ 与 $\mathbf{1 b}$}

将片段 $1 \mathrm{a}(1.0 \mathrm{mmol} / \mathrm{L})$ 溶于反应缓冲液 $(6.0 \mathrm{~mol} / \mathrm{L}$ $\mathrm{Gn} \cdot \mathrm{HCl}, 0.2 \mathrm{~mol} / \mathrm{L} \mathrm{Na}_{2} \mathrm{HPO}_{4}, \mathrm{pH}$ 2.6)中, 经亚硝酸钠氧 化 $\left(-10{ }^{\circ} \mathrm{C}\right)$ 后得到 $1 \mathrm{a}$ 的酰基叠氮产物. 再外加硫醇、 颈基乙酸甲酯(MTG), 调节 $\mathrm{pH}$ 至 5.5, 得到 $\mathbf{1 a}$ 的硫酯产 物. 最后加入 $\mathbf{1 b}(1.2 \mathrm{mmol} / \mathrm{L})$, 调节 $\mathrm{pH}$ 至 $6.8 \sim 6.9$, 使 用 HPLC 监测发现 $8 \mathrm{~h}$ 后可得到转化较完全的连接产物 1d. 分离、纯化后获得纯品 $2.1 \mathrm{mg}$ (产率 32\%)(图 3). ESI-MS $m / z: 6550.27[\mathrm{M}+\mathrm{H}]^{+}$.

NEDD8 (76 amino acids)

(a) M(Nle1)LIKVKTLTGKEIEIDIEPTDKVERIKERVEEKEG(A35)IPPQQQRLIYSGKQ M(Nle49)NDEKTAADYKILGGSVLHLVLALRGG

(b)

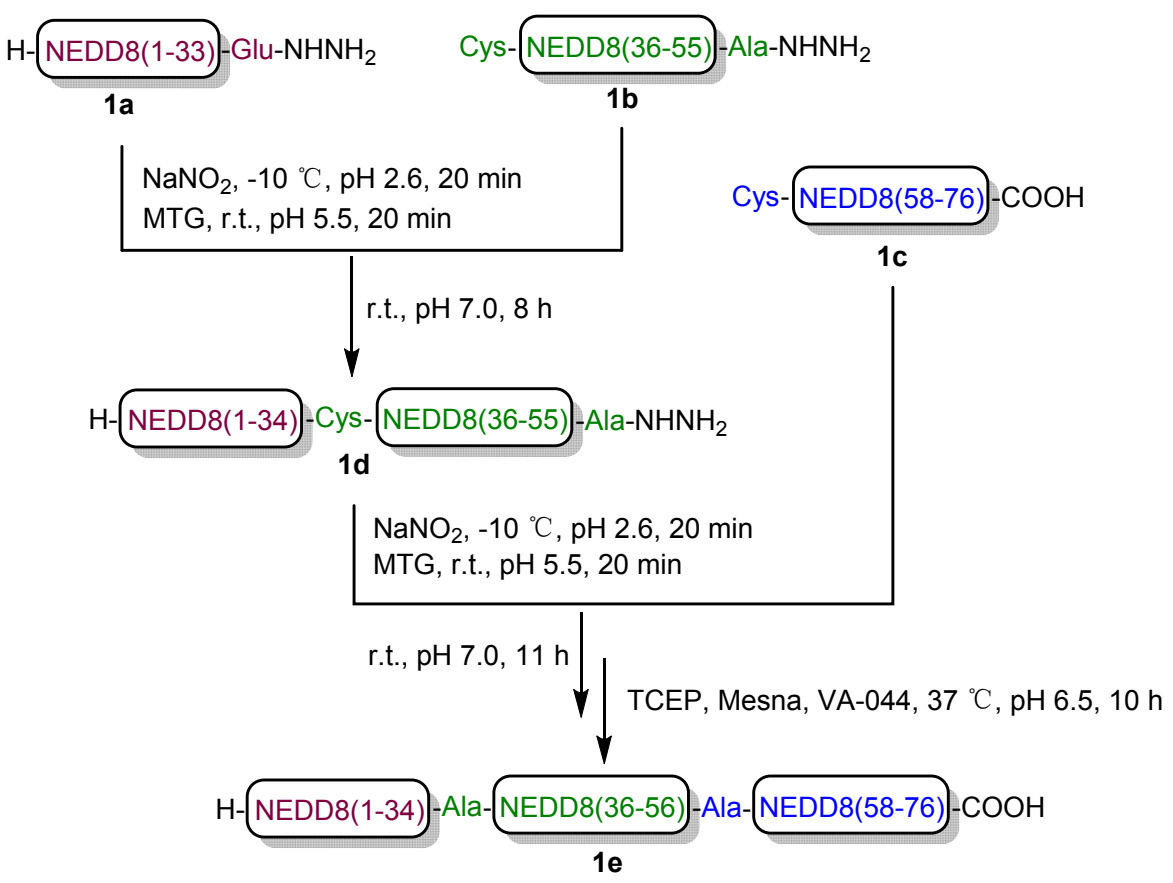

图 1 三片段合成策略

Figure 1 Strategy for three segments synthesis 

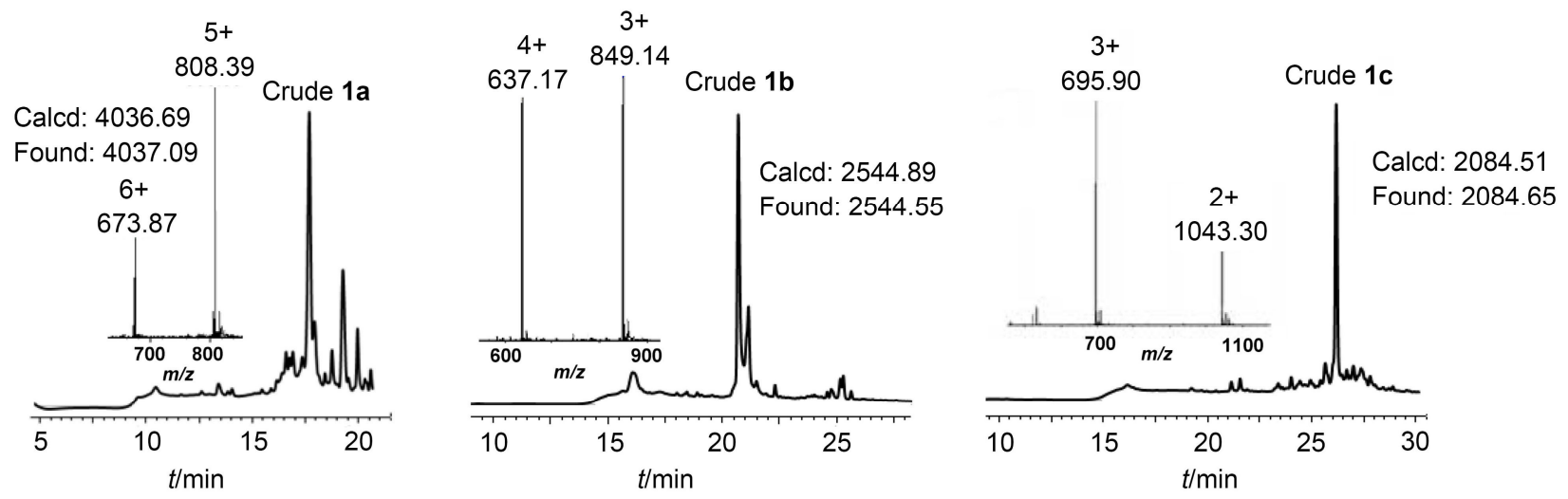

图 2 三片段粗肽的色谱、质谱分析

Figure 2 HPLC and ESI analysis for three segments of crude peptides

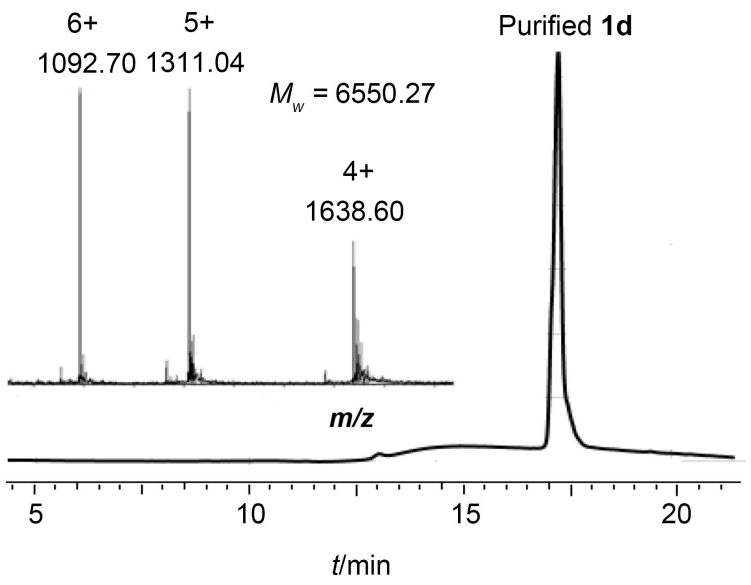

图 3 片段 1d 的色谱和质谱分析

Figure 3 HPLC and ESI-MS analysis of purified peptide 1d

\subsection{3 多肽酰肼法连接片段 $1 \mathrm{c}$ 与 $1 \mathrm{~d}$}

将片段 $1 d$ 溶于 $\mathrm{pH} 2.6$ 的反应缓冲液中, 经亚硝酸 钠氧化 $\left(-10{ }^{\circ} \mathrm{C}\right)$ 后再加入 $\mathrm{MTG}$ 并调节 $\mathrm{pH}$ 至 5.5, 得到 $1 \mathbf{d}$ 的硫酯产物. 此时将片段 1c 加入反应液中, 调节 $\mathrm{pH}$ 至 $6.8 \sim 6.9$. 使用 HPLC 对反应进行监测发现 $11 \mathrm{~h}$ 后即 可得到转化完全的连接产物. 此时在反应液中先后加入 三(2-甲酰乙基)膦盐酸盐(TCEP)、2-颈基乙烷磺酸钠 (Mesna)后调 $\mathrm{pH}$ 至 6.5 , 最后加入偶氮二异丁咪唑啉盐 酸盐(VA-044)进行脱硫. 经纯化、分离后获得全长的 NEDD8 蛋白 1e 约 $0.9 \mathrm{mg}$ (产率 10.5\%)(图 4). ESI-MS $m / z: 8537.58[\mathrm{M}+\mathrm{H}]^{+}$.

\section{2 两片段连接一锅连接脱硫合成 NEDD8}

1.2.1 使用高温辅助 Fmoc-SPPS 法合成 NEED8 片段 虽然通过三片段连接策略可以获得全长 NEDD8 蛋 白, 但多次连接、纯化过程不仅操作繁琐也使得产率并 不理想. 近期本课题组发展的高温辅助 Fmoc-SPPS 方 法，该策略有助于合成序列更长、纯度更高的肽片段 ${ }^{[12]}$. 基于此，我们首先尝试合成长片段 NEDD8(1-56) (2a),

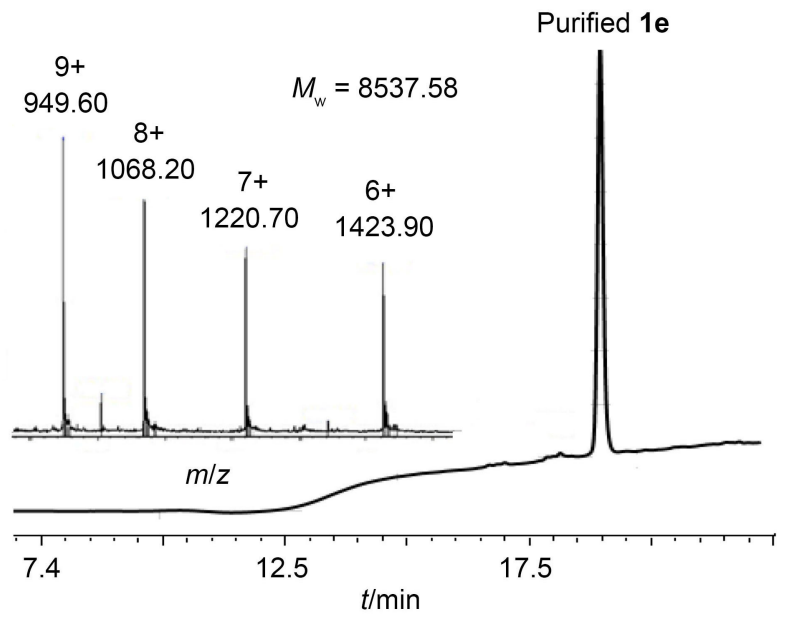

图 4 全长 NEDD8 (1e)纯化后的色谱和质谱分析 Figure 4 HPLC and ESI-MS analysis of full length purified NEDD8 (1e)

进而通过两片段连接的获得蛋白，并结合基于 MTG 的 一锅连接脱硫方法提高合成效率(图 5) ${ }^{[13]}$. HPLC 及质谱 结果证明, 通过使用高温辅助 Fmoc-SPPS 策略两段肽 均可以正确获得, 尤其是片段 $\mathbf{2 a}$ 的合成效率也较为理 想. 经分离、纯化后得到纯品 $\mathbf{2 a} 13.5 \mathrm{mg}$ (分离效率 11.3\%)及 2b $18.6 \mathrm{mg}$ (分离产率 30.5\%) (图 6).

\subsection{2 一锅连接脱硫获得全长 NEDD8 (2c)}

在获得片段 $2 \mathbf{a}$ 和 $\mathbf{2 b}$ 后, 我们首先将其连接以获得 全长蛋白. 将 $\mathbf{2 a}$ 溶于 $\mathrm{pH} 2.6$ 的反应缓冲液中, 经亚硝酸 钠化再加入 $\mathrm{MTG}$ 后调 $\mathrm{pH}$ 至 5.5, 可得到 $\mathbf{2 a}$ 的硫酯产物. 此时将片段 $\mathbf{2 b}$ 加入到反应液里，调节 $\mathrm{pH}$ 至 $6.8 \sim 6.9$, 使用 HPLC 监测发现 $11 \mathrm{~h}$ 后即可完全获得连接产物. 此 时不经纯化直接在反应液中加入 TCEP、Mesna 后调 $\mathrm{pH}$ 至 6.5, 最后加入 VA-044 进行脱硫, $37{ }^{\circ} \mathrm{C}$ 反应约 $10 \mathrm{~h}$ 即 可得到终产物 2c. 经分离、纯化后获得纯品约 $1.2 \mathrm{mg}$ (产 率 24\%). 我们最终通过两片段连接一锅脱硫策略获得 了天然的全长 NEDD8 蛋白(图 7). ESI-MS $m / z: 8525.67$ 


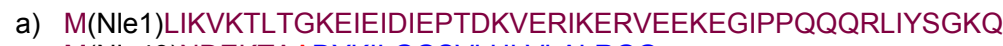
M(Nle49)NDEKTAADYKILGGSVLHLVLALRGG

b)

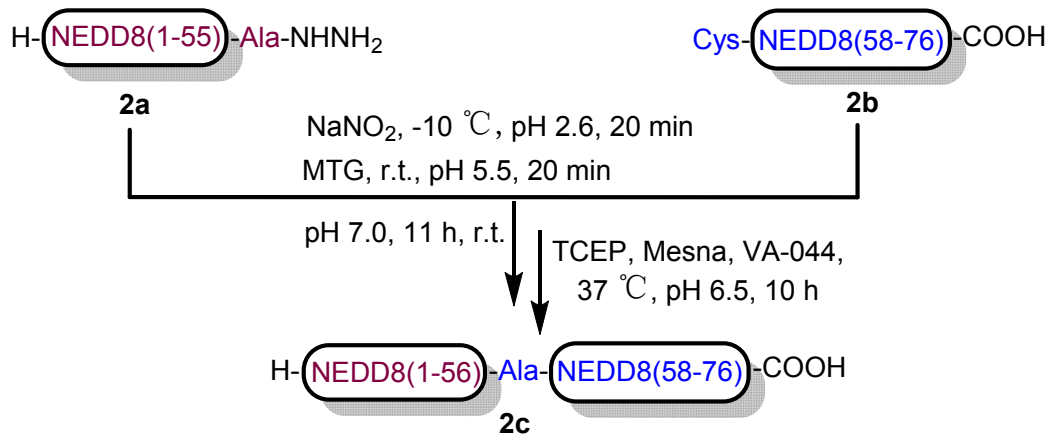

图 5 两片段合成策略

Figure 5 Strategy for one-pot two segments ligation-desulfurization strategy
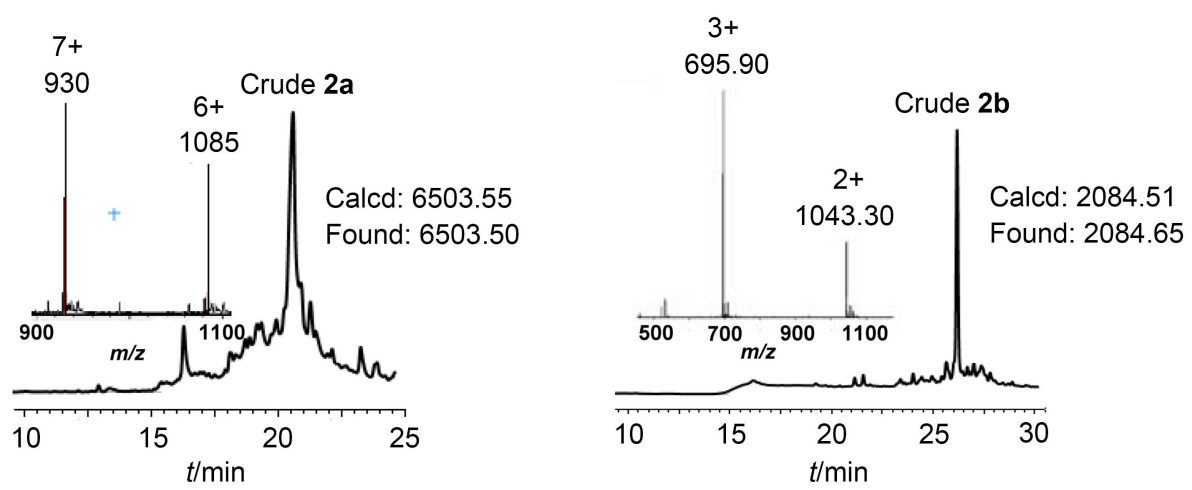

图 6 粗肽片段 $2 a 、 2 b$ 的色谱和质谱分析

Figure 6 HPLC and ESI-MS analysis of crude $\mathbf{2 a}$ and $\mathbf{2 b}$

$[\mathrm{M}+\mathrm{H}]^{+}$.

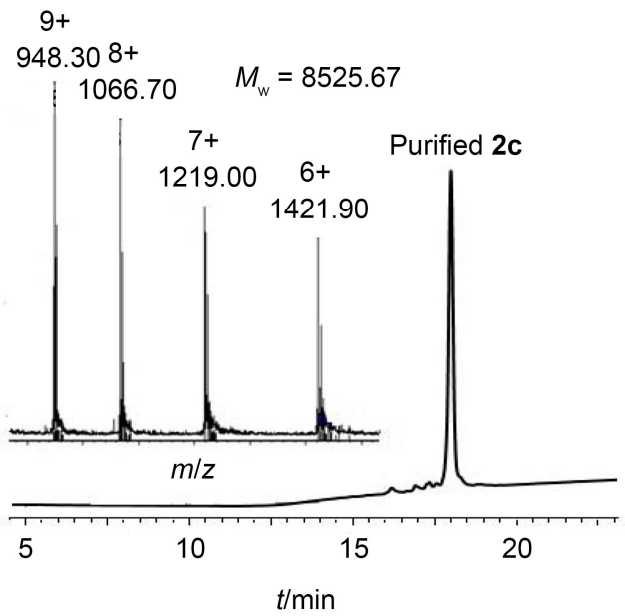

图 7 两片段合成全长 NEDD8 的色谱和质谱分析

Figure 7 HPLC and ESI-MS analysis of full length purified NEDD8 via one-pot two-segment ligation-desulfurization

\section{3 合成 NEDD8 与表达 NEDD8 的结构比较}

为了进一步确定合成 NEDD8 的结构正确性, 我们 使用 SDS-PAGE 及圆二色谱(CD)方法将其与生物表达
的 NEDD8 进行了比对. 结果显示, 我们得到了具有较 高均一性的蛋白，与生物表达的 NEDD8 具有类似的二 级结构(图 8).

\section{2 结论}

本研究结合高温辅助 Fmoc-SPPS、多肽酰肼连接及 一锅连接脱硫策略较为高效地实现了重要翻译后修饰 物蛋白 NEDD8 的三片段及两片段的化学合成. 通过与 生物表达 NEDD8 的分析比较, 证明了合成蛋白具有较 好的均一性及正确的二级结构. 本研究为获得大量、均 一性好的 NEDD8 及其他类泛素蛋白提供了一种新途径, 进而为研究 NEDD 化蛋白的机构与功能机制提供了基 础.

\section{3 实验部分}

\section{1 仪器与试剂}

分析及半制备型 HPLC 使用岛津(Prominence LC20AT), 分析型色谱柱使用 Grace Vydac “Peptide C18”, $150 \mathrm{~mm} \times 4.6 \mathrm{~mm}$, 流速为 $1.0 \mathrm{~mL} / \mathrm{min}$, 紫外检测器波长 为 214 和 $254 \mathrm{~nm}$. 半制备型色谱柱使用 Grace Vydac 


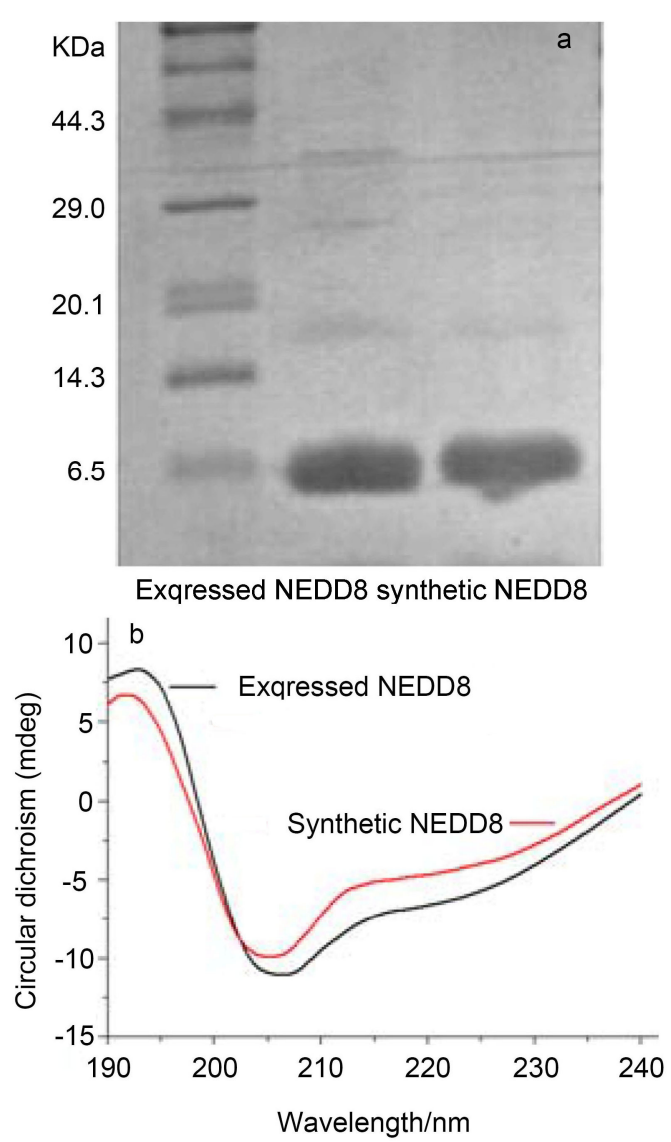

图 8 SDS-PAGE 及 CD 分析比较合成与表达 NEDD8

Figure 8 Comparison of synthetic NEDD8 and expressed NEDD8 via SDS-PAGE and CD analysis

“Peptide C18”, $250 \mathrm{~mm} \times 10 \mathrm{~mm}$, 流速为 $4.0 \mathrm{~mL} / \mathrm{min}$, 紫外检测器波长为 214 和 $254 \mathrm{~nm}$. 流动相 $\mathrm{A}$ 使用含有 $0.08 \%$ 三氟乙酸(TFA)的乙腈, 流动相 B 使用含有 $0.1 \%$ TFA 的水. 分析型 HPLC 使用乙腈浓度为 $10 \% \sim 90 \%$ 及 $20 \% \sim 80 \%$ 的梯度洗脱，梯度时间分别为 30 和 $20 \mathrm{~min}$. 半制备型 HPLC 使用乙腈浓度为 $10 \%$ ～90\%和 $20 \%$ $80 \%$ 的梯度洗脱, 梯度时间均为 $30 \mathrm{~min}$. 除特殊说明所 采用试剂均为市售分析纯.

\section{2 实验方法}

\subsection{1 酰肼树脂的制备}

称取 $232 \mathrm{mg}(0.1 \mathrm{mmol}) 2-\mathrm{Cl}-\operatorname{Trt}(\mathrm{Cl})$ 树脂(取代度为 $0.43 \mathrm{mmol} / \mathrm{g}$ )于固相合成管内, 将 $N, N$-二甲基甲酰胺 $(\mathrm{DMF})$ 与二氯甲烷 $(\mathrm{DCM})$ 按照 $(V: V=1: 1)$ 的比例加入 到合成管内(混合液体积没过树脂即可), 将树脂溶胀 10 min 后抽干, 加入含 $5 \% \mathrm{NH}_{2} \mathrm{NH}_{2} \cdot \mathrm{H}_{2} \mathrm{O}$ 的 DMF 溶液 4 $\mathrm{mL}$, 室温反应两次每次 $1 \mathrm{~h}$. 反应结束后加入 $4 \mathrm{~mL}$ 甲醇 继续反应 $30 \mathrm{~min}$. 最后按照 DMF, DCM, DMF 的顺序各 洗三次, 抽干后即用.

\subsection{2 高温辅助 Fmoc-SPPS}

标准的高温辅助 Fmoc-SPPS 各试剂的用量为: 4 equiv.氨基酸，4.5 equiv. 2-肜氭乙酸乙酯(Oxyma)，4.5 equiv. $N, N^{\prime}$-二异丙基碳二胺(DIC), 反应前需预先活化 6 $\min$, 反应温度 $90{ }^{\circ} \mathrm{C}$, 反应时长 $20 \mathrm{~min}$. 特殊氨基酸的 反应条件如下, Arg: 4 equiv.氨基酸, 3.6 equiv. 6-氯苯并 三氮唑-1,1,3,3-四甲基脲六氟磷酸酯(HCTU), 8 equiv. $\mathrm{N}, \mathrm{N}$-二异丙基乙胺(DIEA), 室温反应 $50 \mathrm{~min}$; Cys: 4 equiv 氨基酸 4.5 equiv. Oxyma, 4.5 equiv. DIC, 反应前需 预先活化 $6 \mathrm{~min}, 50{ }^{\circ} \mathrm{C}$ 反应 $20 \mathrm{~min}$. 所有序列中的 Met 均用 $\mathrm{Nle}$ 代替, 反应按照标准条件进行. 每个氨基酸反 应结束后用含 $20 \%$ 哌啶的 DMF 溶液哌解 $6 \mathrm{~min}$ 以脱除 Fmoc 保护基，再按照 DMF, DCM, DMF 的顺序各洗 3 4 次. 所有氨基酸连接完成后用 $8 \mathrm{~mL}$ 切割溶液 $[88 \%$ TFA, $5 \% \mathrm{H}_{2} \mathrm{O}, 5 \%$ 苯酚, $2 \%$ 三异丙基硅烷(TIPS)]将多肽 片段从树脂上切除，同时脱除掉所有氨基酸侧链保护 基，脱除时间为 $2 \mathrm{~h}$. 之后将切割液收集至离心管中，通 入氮气浓缩再用冰乙醚沉淀、离心后风干即可得到粗肽. 粗肽经半制备型 HPLC 分离、冻干后得到纯肽.

\subsection{3 多肽酰胖连接}

称取 $1 \mathrm{mmol} / \mathrm{L}$ 的 $\mathrm{C}$ 端为酰胖的多肽片段溶于 $\mathrm{pH}$ 2.6 的反应缓冲液中 $(900 \mu \mathrm{L})$, 在 $-10{ }^{\circ} \mathrm{C}$ 的条件下加入 $5 \mathrm{mmol} / \mathrm{L}$ 的亚硝酸钠溶液至终体积为 $1 \mathrm{~mL}$, 反应 20 $\min$ 后加入 $30 \mathrm{mmol} / \mathrm{L}$ 的硫醇试剂, 调 $\mathrm{pH}$ 至 7.0 , 之后 加入 $1.2 \mathrm{mmol} / \mathrm{L} \mathrm{N}$ 端为 Cys 的多肽片段, 使用分析型 HPLC 监测反应. 反应完成后使用半制备型 HPLC 分离 连接产物, 冻干后即可得到纯品 ${ }^{[16]}$.

\subsection{4 基于 $\mathrm{MTG}$ 的一锅连接-脱硫策略}

称取 $1 \mathrm{mmol} / \mathrm{L}$ 的 $\mathrm{C}$ 端为酰肼的多肽片段溶于 $\mathrm{pH}$ 2.6 的反应缓冲液中 $(900 \mu \mathrm{L})$, 在 $-10{ }^{\circ} \mathrm{C}$ 的条件下加入 $5 \mathrm{mmol} / \mathrm{L}$ 亚硝酸钠溶液至终体积为 $1 \mathrm{~mL}$, 反应 $20 \mathrm{~min}$ 后加入 $30 \mathrm{mmol} / \mathrm{L}$ 的 $\mathrm{MTG}$ 并调 $\mathrm{pH}$ 至 5.5, 反应 $20 \mathrm{~min}$ 后加入 $1.2 \mathrm{mmol} / \mathrm{L} \mathrm{N}$ 端为 $\mathrm{Cys}$ 的多肽片段, 使用分析型 HPLC 监测反应. 反应完成后向反应液里先后加入 250 $\mathrm{mmol} / \mathrm{L}$ 的 TCEP, $100 \mathrm{mmol} / \mathrm{L}$ 的 Mesna，调节 $\mathrm{pH}$ 至 6.5, 最后加入 $30 \mathrm{mmol} / \mathrm{L}$ 的 VA-044, 在 $37{ }^{\circ} \mathrm{C}$ 下进行脱硫反 应约 $10 \mathrm{~h}$. 反应完成后使用半制备型 HPLC 分离连接产 物, 冻干后即可得到纯品.

辅助材料(Supporting Information) 各个肽片段色谱 与质谱的原始谱图. 这些材料可以免费从本刊网站 (http://sioc-journal.cn/)上下载.

\section{References}

[1] (a) Pickart, C. M.; Eddins, M. J. Biochim. Biophys. Acta 2004, $1695,55$.

(b) Pickart, C. M.; Fushman, D. Curr. Opin. Chem. Biol. 2004, 8, 610 . 
[2] (a) Matunis, M. J.; Coutavas, E.; Blobel, G. J. Cell Biol. 1997, 135, 1457.

(b) Kumar, S.; Yoshida, Y.; Noda, M. Biochem. Biophys. Res. Commun. 1993, 195, 393.

[3] (a) Hochstrasser, M. Cell 2006, 124, 27. (b) Pickart, C. M. Cell 2004, 116, 181.

[4] Pan, Z. Q.; Kentsis, A.; Dias, D. C.; Yamoah, K.; Wu, K. Oncogene 2004, 23, 1985

[5] (a) Duda, D. M.; Borg, L. A.; Scott, D. C.; Hunt, H. W.; Hammel, M.; Schulman, B. A. Cell 2008, 134, 995.

(b) Scott, D. C.; Sviderskiy, V. O.; Monda, J. K.; Lydeard, J. R.; Cho, S. E.; Harper, J. W.; Schulman, B. A. Cell 2014, 157, 1671.

(c) Cavadini, S.; Fischer, E. S.; Bunke, R. D.; Potenza, A.; Lingaraju, G. M.; Goldie, K. N.; Mohamed, W. I.; Faty, M.; Petzold, G.; Beckwith, R. E. J.; Tichkule, R. B.; Hassiepen, U.; Abdulrahman, W.; Pantelic, R. S.; Matsumoto, S.; Sugasawa, K.; Stahlberg, H.; Thomä, N. H. Nature 2016, 531, 598.

[6] Singh, R. K.; Zerath, S.; Kleifeld, O.; Scheffner, M.; Glickman, M. H.; Fushman, D. Mol. Cell. Proteomics 2012, 11, 1595.

[7] (a) Stickle, N. H.; Chung, J.; Klco, J. M.; Hill, R. P.; Kaelin, W. G. Jr.; Ohh, M. Mol. Cell. Biol. 2004, 24, 3251.

(b) Harper, J. Cell 2004, 118, 2.

(c) Xirodimas, D. P.; Saville, M. K.; Bourdon, J. C.; Hay, R. T.; Lane, D. P. Cell 2004, 118, 83.

(d) Xirodimas, D. P.; Sundqvist, A.; Nakamura, A.; Shen, L.; Botting, C.; Hay, R. T. EMBO Rep. 2008, 9, 280.

(e) Broemer, M.; Tenev, T.; Rigbolt, K. T. G.; Hempel, S.; Blagoev, B.; Silke, J.; Ditzel, M.; Meier, P. Mol. Cell 2010, 40, 810.

(f) Benjamin, S.; Steller, H. Dev. Cell 2010, 19, 791.

[8] (a) Xirodimas, D. P.; Sundqvist, A.; Nakamura, A.; Shen, L.; Botting, C.; Hay, R. T. EMBO Rep. 2008, 9, 280.

(b) Jones, J.; Wu, K.; Yang, Y. Y.; Guerrero, C.; Nillegoda, N.; Pan, Z. Q.; Lan, H. J. Proteome Res. 2008, 7, 1274.

[9] Jbara, M.; Maity, S. K.; Seenaiah, M.; Brik, A. J. Am. Chem. Soc. 2016, $138,5069$.

[10] (a) Kent, S. B. H. Chem. Soc. Rev. 2009, 38, 338.

(b) Raibaut, L.; Ollivier, N.; Melnyk, O. Chem. Soc. Rev. 2012, 41, 7001 . (c) Huang, Y. C.; Liu, L. Sci. China Chem. 2015, 58, 1779.

(d) Huang, Y. C.; Fang, G. M.; Liu, L. Natl. Sci. Rev. 2016, 3, 107.

(e) Liu, H.; Li, X. C. Org. Biomol. Chem. 2014, 12, 3768.

[11] (a) Fang, G. M.; Li, Y. M.; Huang, Y. C.; Li, J. B.; Cui, H. K.; Liu, L. Angew. Chem., Int. Ed. 2011, 50, 7645.

(b) Fang, G.-M.; Wang, J.-X.; Liu, L. Angew. Chem., Int. Ed. 2012, $51,10347$.

(c) Tang, S.; Si, Y. Y.; Wang, Z. P.; Mei, K. R.; Chen, X.; Cheng, J. Y.; Zheng, J.-S.; Liu, L. Angew. Chem., Int. Ed. 2015, 54, 5713.

(d) Wang, J. X.; Fang, G. M.; He, Y.; Qu, D. L.; Yu, M.; Hong, Z. Y.; Liu, L. Angew. Chem., Int. Ed. 2015, 54, 2194.

(e) Zheng, J. S.; Yu, M.; Qi, Y. K.; Tang, S.; Shen, F.; Wang, Z. P.; Xiao, L.; Zhang, L.; Tian, C. L.; Liu, L. J. Am. Chem. Soc. 2014, 136,3695 .

(f) Zheng, J.-S.; He, Y.; Zuo, C.; Cai, X.-Y.; Tang, S.; Wang, Z. A.; Zhang, L.-H.; Tian, C.-L.; Liu, L. J. Am. Chem. Soc. 2016, 138, 3553 .

[12] Huang, Y. C.; Guan, C. J.; Tan, X. L.; Chen, C. C.; Guo, Q. X.; Li, Y. M. Org. Biomol. Chem. 2015, 13, 1500.

[13] Huang, Y. C.; Chen, C. C.; Gao, S.; Wang, Y. H.; Xiao, H.; Wang, F.; Tian, C. L.; Li, Y. M. Chem. Eur. J. 2016, 22, 7623.

[14] Kochendoerfer, G. G.; Kent, S. B. H. Curr. Opin. Chem. Biol. 1999, 3,665 .

[15] (a) Miller, M.; Schneider, J.; Sathyanarayana, B. K.; Toth, M. V.; Marshall, G. R.; Clawson, L.; Selk, L.; Kent, S. B. H.; Wlodawer, A. Science 1989, 246, 1149.

(b) Deng, F. K.; Zhang, L.; Wang, Y. T.; Schneewind, O.; Kent, S. B. H. Angew. Chem., Int. Ed. 2014, 53, 4662.

[16] (a) Zheng, J. S.; Tang, S.; Huang, Y. C.; Liu, L. Acc. Chem. Res. 2013, 46, 2475.

(b) Zheng, J. S.; Tang, S.; Qi, Y. K.; Wang, Z. P.; Liu, L. Nat. Protoc. 2013, 8, 2483.

(c) Li, J. B.; Li, Y. Y.; He, Q. Q.; Li, Y. M.; Li, H. T.; Liu, L. Org. Biomol. Chem. 2014, 12, 5435.

(d) Chen, C. C.; Li, S. J.; Chen, Y. Q.; Xu, H. J.; Li, Y. M. Chin. J. Org. Chem. 2014, 34, 1452.

(e) Li, Y. M.; Li, Y. T.; Pan, M.; Kong, X. Q.; Huang, Y. C.; Hong, Z. Y.; Liu, L. Angew. Chem., Int. Ed. 2014, 53, 2198.

(Li, L.; Fan, Y.) 